\title{
Collective Entrainment and Confinement Amplify Transport by Schooling Microswimmers
}

\author{
Chenyu Jin ${ }_{1}^{1,2}$ Yibo Chen $\odot,{ }^{3}$ Corinna C. Maass $\odot,{ }^{2,3, *}$ and Arnold J. T. M. Mathijssen $\odot^{4, \dagger}$ \\ ${ }^{1}$ Experimentalphysik I, Universität Bayreuth, Bayreuth 95440, Germany \\ ${ }^{2}$ Max Planck Institute for Dynamics and Self-Organization and Institute for the Dynamics of Complex Systems, \\ Georg August Universität, 37077 Göttingen, Germany \\ ${ }^{3}$ Physics of Fluids Group, Max Planck Center for Complex Fluid Dynamics, \\ MESA+ Institute and J. M. Burgers Center for Fluid Dynamics, University of Twente, \\ PO Box 217,7500 AE Enschede, The Netherlands \\ ${ }^{4}$ Department of Physics \& Astronomy, University of Pennsylvania, \\ 209 South 33rd Street, Philadelphia, Pennsylvania 19104, USA
}

(Received 5 April 2021; accepted 19 July 2021; published 20 August 2021)

\begin{abstract}
Microswimmers can serve as cargo carriers that move deep inside complex flow networks. When a school collectively entrains the surrounding fluid, their transport capacity can be enhanced. This effect is quantified with good agreement between experiments with self-propelled droplets and a confined Brinkman squirmer model. The volume of liquid entrained can be much larger than the droplet itself, amplifying the effective cargo capacity over an order of magnitude, even for dilute schools. Hence, biological and engineered swimmers can efficiently transport materials into confined environments.
\end{abstract}

DOI: 10.1103/PhysRevLett.127.088006

"At low Reynolds number you can't shake off your environment. If you move, you take it along" [1]. This principle, that a moving particle permanently displaces its surrounding fluid, was quantified in 1953 by Darwin [2,3]. The amount of liquid entrained, the "Darwin drift volume," diverges for a colloid pulled along an infinite straight line through an unconfined Stokesian fluid [4,5], also at intermediate Reynolds numbers [6]. However, the drift volume is finite for microswimmers that do not exert a net force on the liquid [7,8]. Hydrodynamic entrainment can be a curse and a blessing: it is important in a wide range of biological and ecological processes, including enhanced diffusion [9-18], biogenic mixing [19-24], food uptake [25-29], particle transport [30-34], fungal spore dispersal [35], oxygen redistribution [36], and microbial interaction probabilities [37].

Here, we consider collective entrainment by a school (flock, or swarm) of microswimmers. Beyond biological systems this could equally be advantageous for microrobotic material transport, especially in confined environments. A wide range of synthetic microswimmers has been developed in recent years, including active Janus colloids, magnetic swimmers, and bimetallic nanorods [38-45]. While these swimmers could serve as carriers that transport a payload deep into a network of microchannels, their internal cargo space is inherently limited [46-54]. Therefore, instead of transporting cargo inside the microswimmer, we consider entraining the medium outside it, so that passive cargo vesicles or compounds dissolved in the fluid itself can be pushed forward by groups of swimmers cooperatively. To investigate this quantitatively, we focus on self-propelled droplets $[55,56]$, which feature a (a)
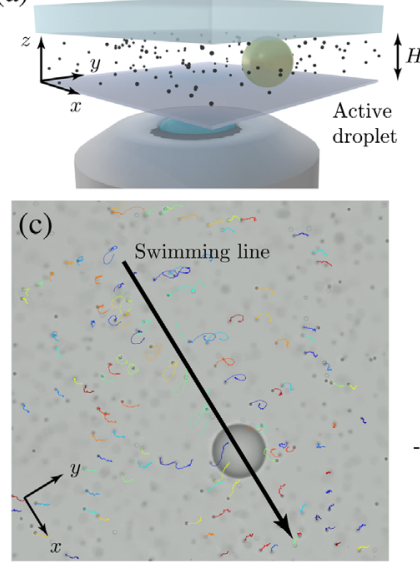

(b)
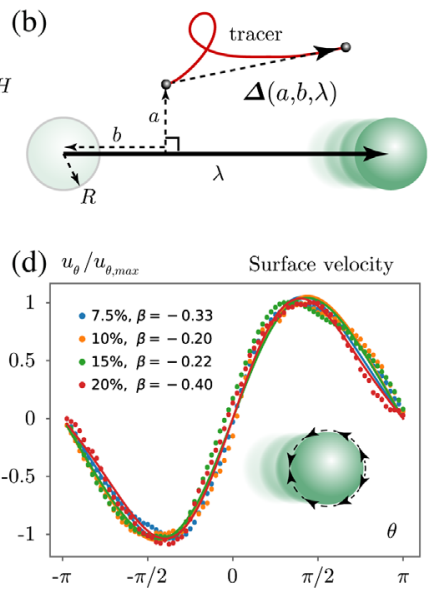

FIG. 1. Fluid transport by a self-propelled droplet. (a) Schematic of experimental setup. Cell bottom glass, top PDMS, $H=52 \mu \mathrm{m}$, droplet radius $R=24 \mu \mathrm{m}$. (b) Diagram of an entrainment event. Because of the flow generated by the droplet, the tracer is displaced by $\Delta(a, b, \lambda)$ as a function of the impact parameters $a$ and $b$, and the swimming path length $\lambda$. (c) Movie screenshot showing particle trajectories due to an active droplet swimming along a straight line (see Video 1). (d) Tangential flow velocity at the surface of the droplet, in the comoving frame of reference. Data points are PIV measurements close to the interface, for four different surfactant concentrations. Lines are fits to the first two modes of the squirmer model (Eq. (1)). Legend: resulting dipole coefficient $\beta=B_{2} / B_{1}$ for each TTAB concentration. 
well-developed toolbox for tuning their motility, flow generation, and collective dynamics, and solving microfluidic mazes [57-68].

We begin by collecting data on the dynamics of tracer particles displaced by self-propelling droplets. Our experimental methods are described in Sec. I of the Supplemental Material [69]. In our setup [Fig. 1(a)] we place a (S)-4Cyano-4'-(2-methylbutyl)biphenyl (CB15) oil droplet of radius $R=24 \mu \mathrm{m}$ in quasi-2D confinement of a polydimethylsiloxane (PDMS)-glass reservoir with $H=52 \mu \mathrm{m}$, where it self-propels with a speed of $U=(21.8 \pm 0.5) \mu \mathrm{ms}^{-1}$. The swimming medium is a micellar aqueous solution of tetradecyltrimethylammonium bromide (TTAB) surfactant at 5\% mass fraction, containing a low density $\left(\approx 10^{5} / \mu \mathrm{l}\right)$ of polystyrene tracer colloids with diameter $d_{C}=2 \mu \mathrm{m}$. Heavy water is added to the swimming medium in order to match its density with the tracer colloids. The droplet propulsion is powered by a selfsupporting interfacial surface tension gradient $[56,85]$. Using video microscopy, we extract trajectories for approximately 300-400 tracer colloids [Figs. 1(b) and 1(c) and Video 1] whose $z$ position did not deviate too far from the chamber's midplane during the transit of the active droplet. After correcting a slight background drift, all coordinates are translated and rotated such that the swimmer moves along the $x$ axis. Hence, we determine the displacement of a tracer particle, $\Delta(a, b, \lambda)$, following the notation by Lin et al. [13], where the impact parameters $a, b$ specify the initial perpendicular and parallel distance of the tracer to the start of the swimming path, and $\lambda$ is the swimming path length [Fig. 1(b)]. The droplets move persistently, with $\lambda \approx 100 R$ before they reorient due to rotational fluctuations, likely from inhomogeneities in the surfactant coverage.

We then seek to describe the flow $\boldsymbol{u}(\boldsymbol{r})$ produced by the droplet due to its self-propulsion. Using particle image velocimetry (PIV), we first measure the tangential flow velocity $u_{\theta}$ near the droplet interface [Fig. 1(d), points]. These experiments are compared with the squirmer model $[86,87]$, where the surface velocity is given by the mode decomposition

$$
\left.u_{\theta}\right|_{r=R}=B_{1} \sin (\theta)+\frac{1}{2} B_{2} \sin (2 \theta)+\ldots
$$

By fitting this model to our data, we find the mode strengths $B_{1}=(29.8 \pm 2.0) \mu \mathrm{ms}^{-1}$ and $B_{2}=(-7.8 \pm 2.0) \mu \mathrm{ms}^{-1}$. Using only these first two modes already offers a good agreement with the measured flow [Fig. 1(d), lines]. The dipole coefficient is $\beta=B_{2} / B_{1} \approx-0.3$, so the droplets are weak pushers. These experiments are repeated for four different TTAB concentrations [Fig. 1(d), legend].

The measured flows generated by an active droplet are shown in the top halves of Figs. 2(a) and 2(b). These experiments agree well with the results by De Blois et al. [64], showing strong regions of forward flow (red colors in
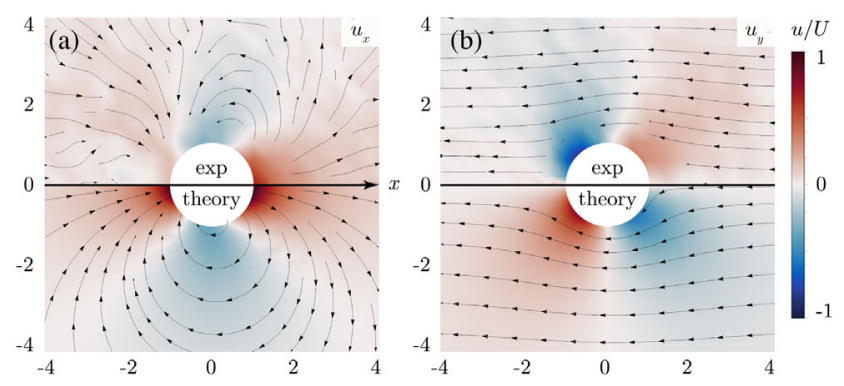

(c) $\quad\left|u_{x}\right| / U$ along $y=0$

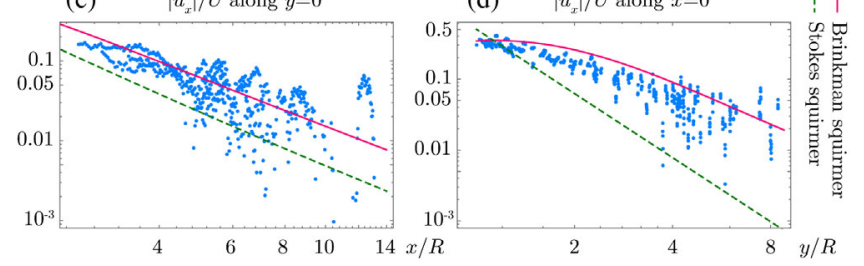

FIG. 2. Flows $\boldsymbol{u}(\boldsymbol{r})$ generated by an active droplet. (a),(b) Comparison of the flow fields between (top half) experiments and (bottom half) the squirmer model in a Brinkman fluid (Supplemental Material, Eq. S13). Flows are shown at the midplane of the microfluidic chamber. Streamlines are shown (a) in the laboratory frame, and (b) in the frame comoving with the droplet. Background colors denote (a) the longitudinal flow $u_{x}$ and (b) the transverse flow $u_{y}$, scaled with the swimming speed. Note that the transverse flow $u_{y}$ changes sign across the $x$ axis. (c),(d) Decay of the longitudinal flow strength in the laboratory frame along the (c) $x$ axis and (d) $y$ axis. Points show experiments, and the solid line is the corresponding squirmer model in a Brinkman fluid. The dashed line shows the squirmer model in an unconfined Stokesian fluid, which underestimates the flow strengths.

panel a). This fluid motion leads to entrainment, pushing particles in front of the droplet or dragging them along behind. Quantitatively, however, the measured flow [Figs. 2(c) and 2(d), blue points] is about 5 times stronger than the prediction from the conventional squirmer model in a 3D unconfined Stokesian fluid (green dashed lines).

To provide a more accurate theoretical description of the flows created by an active droplet (Supplemental Material, Sec. II), a squirmer model was developed that accounts for the quasi-2D confinement using the Brinkman equations [88]. The quintessential difference between the conventional Stokes model and the Brinkman model is the confinement, which is described by a permeability parameter in terms of the microfluidic chamber height, $k=12 / H^{2}$, analogous to Darcy's law. The Brinkman squirmer model is then derived by imposing the same tangential velocity at the droplet interface (Eq. (1)) as the boundary condition. The resulting expression (Supplemental Material, Eq. S13) gives an accurate description of the measured flows in all directions [Figs. 2(a) and 2(b), bottom halves]. Potential sources of experimental error are tracers having a small velocity component out of the focal plane, the difficulty of sampling 
near the droplet interface, and the droplet diameter being a bit smaller than the channel height. Therefore, the mean experimental flows are slightly weaker than modeled. Still, compared with the squirmer model in an unconfined Stokesian fluid, the Brinkman model offers a significantly improved agreement [Figs. 2(c) and 2(d), pink lines].

Having quantified the flows made by active droplets, we consider how these currents displace tracer particles (or equivalently, the fluid itself) along the swimming direction. The tracers' equation of motion is

$$
\frac{d \boldsymbol{r}_{T}}{d t}=\boldsymbol{u}\left(\boldsymbol{r}_{T}-\boldsymbol{r}_{S}\right)+\sqrt{2 D} \boldsymbol{\xi}(t),
$$

where $\boldsymbol{r}_{S}(t)$ is the moving droplet position, the flow $\boldsymbol{u}$ is given by Supplemental Material, Eq. S13, $\boldsymbol{\xi}$ is standard white noise, and the particle diffusivity, $D=0.22 \mu \mathrm{m}^{2} / \mathrm{s}$, is determined experimentally by analyzing their Brownian motion in the absence of droplets. The particle displacement is then defined as $\boldsymbol{\Delta}=\boldsymbol{r}_{T}\left(t_{f}\right)-\boldsymbol{r}_{T}\left(t_{i}\right)$, where $t_{i}=0$ and $t_{f}=\lambda / U$.

To illustrate the impact of fluid deformation by a selfpropelled droplet, following Pushkin et al. [7], we first simulate the Brinkman model in the absence of noise. Initially, the fluid parcels are arranged along a square grid, after which the droplet swims through [Fig. 3(a) and Video 2]. The horizontal curtains (initially the same $y$ values) are pushed outward in front of the drop and pulled inward behind it. The vertical curtains are folded around the droplet, compressed in front and stretched out behind the drop. In the middle of the swimming path, $x=0$, particles are displaced backward for large $y$ values [violet trajectory in Fig. 3(a)] but nearby the particles are strongly entrained forward (red trajectory).

The same is observed experimentally [Fig. 3(b)]. To quantify the entrainment, we select particles that are initially located close to the middle of the swimming path, with $\lambda=15 R$ and impact parameter $5<b / R<10$. Far from the droplet, for impact parameters $a \gtrsim 2 R$, the tracers have a final displacement that is backward compared with the swimming direction. Note that it is not always possible to track particles close to the drop because they can move out of the focal plane. Nevertheless, for $a \lesssim 2 R$, we still see a strong forward entrainment.

These measurements are in good agreement with the Brinkman squirmer model [Fig. 3(c)]. Here we simulate particles with Brownian motion (Eq. (2)), where the computed trajectories start from the same initial positions as the experimental particles. Interestingly, these results do not deviate significantly from the deterministic Brinkman model (solid line), obtained from numerically integrating Eq. (2) without Brownian motion and using the same droplet dynamics as in the experiment, which may also be approximated theoretically using asymptotic analysis [89]. However, the transport of particles is substantially different
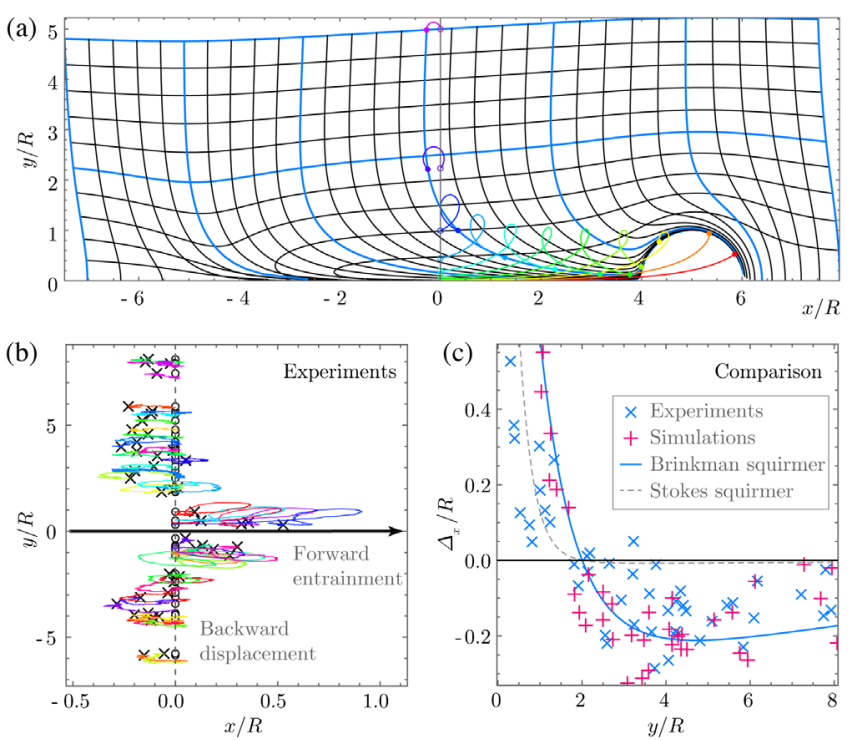

FIG. 3. Entrainment of particles by a confined self-propelled droplet. (a) Fluid transport by a squirmer in a Brinkman fluid. Shown is the deformation of an initially uniform rectangular grid of tracer particles, after a droplet swims from $x / R=-5$ to 5 . Colored trajectories show the motion of particles starting at different positions along the $y$ axis. (b) Experimental trajectories, with their initial positions aligned along the $y$ axis. The initial positions are marked with a (Circle), and the final positions are marked with a (Cross mark). (c) Comparison. (Blue cross mark) show the experimental entrainment along $x$ as a function of lateral distance $y$, (Pink plus signs) show simulated trajectories using the same initial positions using the Brinkman model with Brownian motion, the solid blue line shows the Brinkman theory without noise, and the dashed gray line shows the squirmer model in an unconfined Stokesian fluid. Far away the particles are displaced backward, but nearby they are entrained forward, along the swimming direction.

in bulk liquids (dashed line) compared with confined spaces (solid line).

Next, we consider collective transport by a school of microswimmers, where particles or fluid parcels are pushed forward by multiple subsequent entrainment events [Fig. 4(a) and Video 3]. This principle could apply to a broad range of biological and synthetic microswimmers. Here, we focus on drops that are distributed uniformly in the $x y$ plane with area fraction $\phi$, so the number density is $n=\phi / \pi R^{2}$, and all droplets swim in the $x$ direction with speed $U$. Depending on the kind of microswimmer, this orientation alignment could stem from collective interactions [59,62], internal mechanisms like chemotaxis [61], or guidance by tracks in the microfluidic channel $[65,90]$. Thus, having characterized the entrainment due to a single droplet in the last section, we now simulate tracers that are entrained by a school (see Supplemental Material, Sec. III).

As shown in Fig. 4(b), the individual tracers (gray trajectories) experience large sudden displacement events when a droplet transits nearby (jumps). These events are 

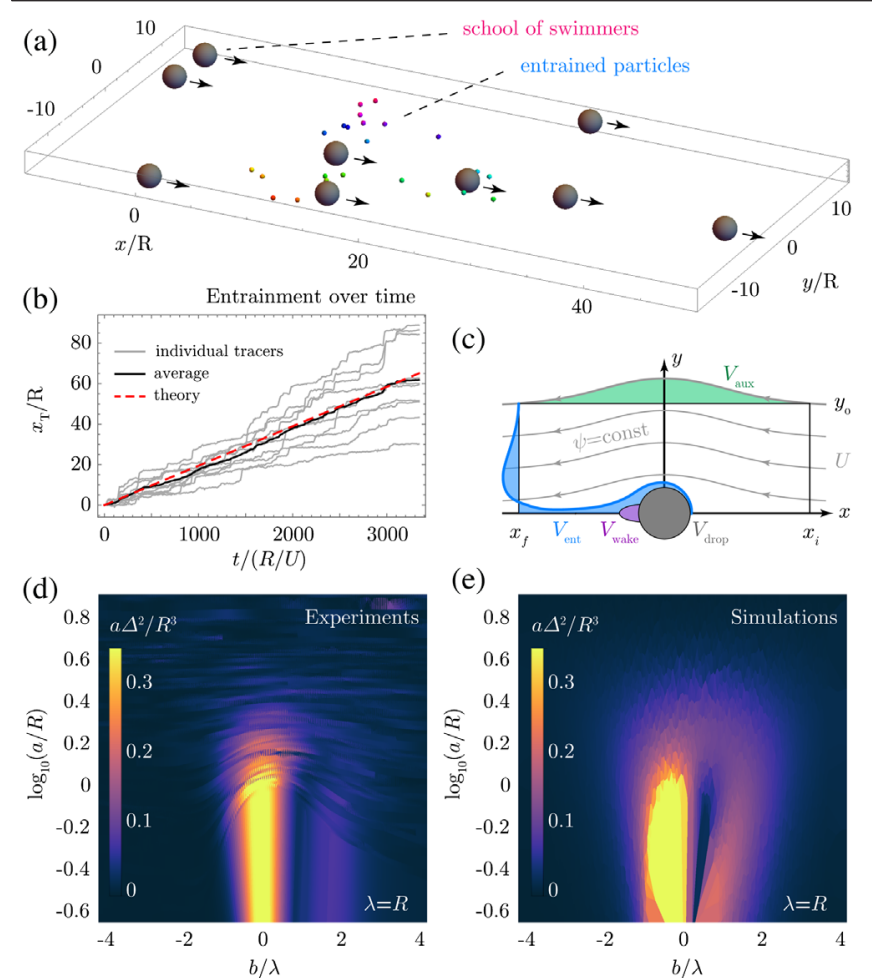

(c)

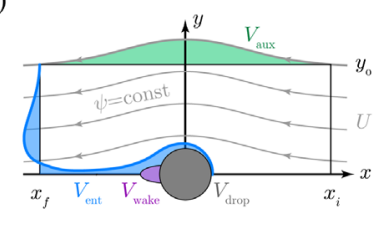

(e)

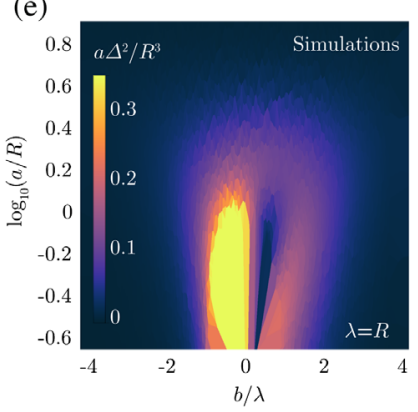

FIG. 4. Entrainment by a school of microswimmers. (a) Simulation snapshot, see Video 3. Self-propelled droplets (black) move collectively along the positive $x$ direction, transporting particles (colors) that initially started at $x=0$. (b) Displacement of the particles along the swimming direction as a function of time, $x_{T}(t)$. Individual trajectories (gray lines) show jumps and drift events, which on average (black line) lead to the entrainment velocity $U_{\text {ent }}$ that is predicted analytically (red dashed line) by Eq. (5). (c) Diagram of the entrainment volume (blue shaded area) that is dragged behind an active droplet, in the comoving reference frame. The gray lines depict stream lines, the purple area is the swimmer wake, and the green area is the auxiliary volume. (d),(e) Stirring by active droplets in a Brinkman fluid. Shown is the integrand $a R^{-3} \Delta^{2}(a, b, \lambda)$ from Eq. (7), measured experimentally and computed numerically.

separated by long periods of comparatively weak Brownian motion and long-ranged flows (drifts). Averaged over time, or equivalently over a statistical ensemble, these jumps and drifts give rise to a mean entrainment velocity,

$$
\boldsymbol{U}_{\mathrm{ent}}=\left\langle\frac{d \boldsymbol{r}_{T}}{d t}\right\rangle
$$

which quantifies how fast the fluid (and all the particles it contains) is transported along the swimming direction [Fig. 4(b), black solid line].

This mean entrainment velocity can be approximated analytically. For a dilute school of swimmers, $\phi \ll 1$, we can write $U_{\text {ent }}=n U A_{\text {ent }}$, where the first two factors encode the encounter rate, and $V_{\text {ent }}=H A_{\text {ent }}$ is the volume of liquid entrained by a single droplet. This is also called the

"Darwin drift" volume [2,7], the region swept out by a tracer curtain [Fig. 4(c), blue region]. Specifically, in our quasi-2D geometry and in the limit of $\lambda \rightarrow \infty$ and $b=\lambda / 2$, the entrainment volume is defined as

$$
V_{\mathrm{ent}}=2 H \int_{0}^{\infty} \Delta_{x} d a
$$

To compute this quantity analytically (Supplemental Material, Sec. III B), we use a stream function formulation to determine the Darwin drift volume in the confined Brinkman medium. Hence, we find that a school of squirmers can transport their surrounding fluid with velocity

$$
\frac{U_{\text {ent }}}{U}=\frac{\phi V_{\text {ent }}}{\pi R^{2} H}=\phi\left[1+\frac{2\left(2 U-B_{1}\right) h K_{1}\left(\frac{R}{h}\right)}{U R K_{0}\left(\frac{R}{h}\right)}\right],
$$

which applies to both pushers and pullers with dipole coefficient $|\beta|<1$. Here $h=(H / \sqrt{12})$ and $K_{n}(x)$ are modified Bessel functions of the second kind. Inserting $\phi=0.01$ and our experimental values into Eq. (5), we obtain the red dashed line in Fig. 4(b), which offers a good agreement with the simulation. The school can generate a significant fluid transport, because all the liquid between the swimmers also moves, with speed $U_{\text {ent }}$ on average. Therefore, a substantial amount of cargo can be moved within the medium, even if $U_{\text {ent }}$ is small compared with $U$. To quantify this, we write the internal transport as $\Theta_{0}=\phi U \eta$, where $\eta \in[0,1]$ denotes the internal storage efficiency, defined as the volume of the cargo held compared to the total volume of the swimmer. In drug delivery this quantity is also called the loading capacity, which is typically $\eta \sim 0.1$ or less [46-54]. Then, the total transport is

$$
\Theta=\phi U \eta+(1-\phi) U_{\mathrm{ent}} .
$$

For an ideal carrier, $\eta=1$, using $\phi=0.01$ and $U_{\text {ent }} \approx$ $0.02 U$ from Eq. (5), the total transport is already tripled by entrainment, $\Theta / \Theta_{0} \approx 3$. Moreover, if the space for cargo inside the swimmer is poor, $\eta=0.1$, the total transport is over 20 times enhanced, $\Theta / \Theta_{0} \approx 20$.

Interestingly, the confinement can help with the particle transport. For a squirmer in an unconfined Stokesian fluid, Pushkin et al. [7] found that entrainment volume is equal to half the droplet volume, $V_{\text {ent }} / V_{\text {drop }}=0.5$, if $|\beta|<1$. In the confined Brinkman medium, we find $V_{\text {ent }} / V_{\text {drop }} \approx 2.93$ (Supplemental Material, Eq. S24), so about 6 times larger. This result is confirmed by integrating the curtain displacement (Eq. (4)) numerically (Supplemental Material, Sec. III C).

Before closing this Letter, we also discuss how swimming droplets can enhance particle diffusion [9-17]. Building on the same framework, we now consider droplets 
without alignment that perform uncorrelated, random reorientations in the $x y$ plane, with swimming path length $\lambda$. Then, the total displacement $\Delta(a, b, \lambda)$ can be used to estimate the stirring efficiency. Combining Equations 2.5 and 3.2 by Lin et al. [13], for a dilute swimmer suspension, the enhanced diffusivity is given by

$$
D_{\mathrm{enh}}=\frac{\phi R U}{2 \pi} \int_{\mathbb{R}^{2}} \frac{a \Delta^{2}(a, b, \lambda)}{R^{3}} d\left(\frac{b}{\lambda}\right) d \log \left(\frac{a}{R}\right) .
$$

Using our particle tracking experiments, we measure the integrand of this expression as a function of the impact parameters $a$ and $b$ [Fig. 4(d)]. These findings compare favorably with the simulated values from the Brinkman squirmer model [Fig. 4(e)]. Hence, by computing the integrand for $a / R \in\left[10^{-2}, 10^{3}\right]$ and $b / \lambda \in[-10,10]$, using $\lambda=100 R$, and integrating these results numerically, we find that the enhanced diffusion is $D_{\text {enh }} / \phi \approx 638 \mu \mathrm{m}^{2} / \mathrm{s}$. Hence, the mixing efficiency can be significantly larger compared with the thermal diffusion of micron-sized particles $\left(D_{\text {th }} \approx 0.2 \mu \mathrm{m}^{2} / \mathrm{s}\right.$ ), even at low droplet densities. We can also compare between active droplets in a quasi-2D fluid and in an unconfined 3D fluid, using the results by Lin et al. [13]. Inserting the parameters of our droplets into their Fig. 6(a) expression, one finds $D_{\text {enh }} / \phi \sim 0.5 n U R^{4} / \phi \sim$ $62 \mu \mathrm{m}^{2} / \mathrm{s}$ in an unconfined fluid. That is about an order of magnitude lower than the quasi-2D result, so the confinement can also amplify particle mixing.

To conclude, we investigated the transport of fluid (or particles embedded therein) by self-propelled droplets. First, by measuring the flows they generate, a Brinkman squirmer model was developed that accounts for the quasi2D confined geometry in a tractable manner. We then quantified the amount of liquid entrained by a single droplet, and we derived the corresponding Darwin drift volume analytically. To reveal how fast a school of droplets can push cargo particles through a microfluidic channel, the transport velocity was computed by integrating over successive entrainment events. Hence, we found that the total cargo capacity can be enhanced significantly compared with transport inside the microcarriers alone. In that sense, we call this phenomenon "collective entrainment," because it relies on the cooperation of a large collection of swimmers, together pushing the cargo forward. The singleswimmer Darwin drift volume already captures the main physics for the average transport velocity in the dilute limit, but the collective transport only arises when the swimmers align with one another in a school.

While this Letter focuses on self-propelled droplets to compare our experiments with the theory, we envisage that these results are widely applicable to other types of microswimmers, particularly active Janus particles [38-45]. The dipole moment could be large for such swimmers, which changes the entrainment volume. Therefore, we extend our discussion of Eq. (5) for the case of $|\beta|>1$ in Supplemental Material, Sec. III E. Interestingly, the entrainment volume is the same for pushers and pullers (Fig. S1). More generally, using the universality of this model, one could account for almost any hydrodynamic signature with higher-order moments. Thus, together with recent developments in microrobotics [50,54], an efficient cargo transport could be established in microfluidic networks or drug delivery applications [46-54].

Besides synthetic active particles, these results could be equally significant for material transport by biological microswimmers $[17,24]$. By directed collective motion through confined spaces [91,92], they could entrain nutrients or signaling molecules deep into complex networks, including biofilm architectures [93], porous media [94], or foams [95], much faster than transport by thermal diffusion. Ultimately, self-propelled particles rely on the replenishment of fuel, particularly into confined spaces, to sustain their activity.

A. M. acknowledges funding from the Human Frontier Science Program (Fellowship LT001670/2017) and the United States Department of Agriculture (USDA-NIFA AFRI Grants No. 2020-67017-30776 and No. 2020-6701532330). C. C. M. and C. J. acknowledge funding from the BMBF/MPG MaxSynBio initiative and the DFG SPP 1726 "Microswimmers."

*c.c.maass@utwente.nl †amaths@upenn.edu

[1] E. M. Purcell, Am. J. Phys. 45, 3 (1977).

[2] C. G. Darwin, Math. Proc. Cambridge Philos. Soc. 49, 342 (1953).

[3] I. Eames, S. E. Belcher, and J. C. R. Hunt, J. Fluid Mech. 275, 201 (1994).

[4] I. Eames, D. Gobby, and S. Dalziel, J. Fluid Mech. 485, 67 (2003).

[5] V. A. Shaik and A. M. Ardekani, Phys. Rev. Fluids 5, 013604 (2020).

[6] N. G. Chisholm and A. S. Khair, Phys. Rev. Fluids 2, 064101 (2017).

[7] D. O. Pushkin, H. Shum, and J. M. Yeomans, J. Fluid Mech. 726, 5 (2013).

[8] N. G. Chisholm and A. S. Khair, Phys. Rev. Fluids 3, 014501 (2018).

[9] X.-L. Wu and A. Libchaber, Phys. Rev. Lett. 84, 3017 (2000).

[10] M. J. Kim and K. S. Breuer, Phys. Fluids 16, L78 (2004).

[11] K. C. Leptos, J. S. Guasto, J. P. Gollub, A. I. Pesci, and R. E. Goldstein, Phys. Rev. Lett. 103, 198103 (2009).

[12] J.-L. Thiffeault and S. Childress, Phys. Lett. A 374, 3487 (2010).

[13] Z. Lin, J.-L. Thiffeault, and S. Childress, J. Fluid Mech. 669, 167 (2011).

[14] D. O. Pushkin and J. M. Yeomans, Phys. Rev. Lett. 111, 188101 (2013) 
[15] A. Morozov and D. Marenduzzo, Soft Matter 10, 2748 (2014).

[16] Y. Peng, L. Lai, Y.-S. Tai, K. Zhang, X. Xu, and X. Cheng, Phys. Rev. Lett. 116, 068303 (2016).

[17] R. Jeanneret, D. O. Pushkin, V. Kantsler, and M. Polin, Nat. Commun. 7, 12518 (2016).

[18] F. Guzmán-Lastra, H. Löwen, and A. J. T. M. Mathijssen, Nat. Commun. 12, 1906 (2021).

[19] K. Katija and J. O. Dabiri, Nature (London) 460, 624 (2009).

[20] G. Subramanian, Curr. Sci. 98, 1103 (2010), https://www .jstor.org/stable/24111768.

[21] J. C. Nawroth and J. O. Dabiri, Phys. Fluids 26, 091108 (2014).

[22] I. A. Houghton, J. R. Koseff, S. G. Monismith, and J. O. Dabiri, Nature (London) 556, 497 (2018).

[23] A. J. Mathijssen, J. Culver, M. S. Bhamla, and M. Prakash, Nature (London) 571, 560 (2019).

[24] L. Ortlieb, S. Rafaï, P. Peyla, C. Wagner, and T. John, Phys. Rev. Lett. 122, 148101 (2019).

[25] V. Magar, T. Goto, and T. J. Pedley, Q. J. Mech. Appl. Math. 56, 65 (2003).

[26] M. B. Short, C. A. Solari, S. Ganguly, T. R. Powers, J. O. Kessler, and R. E. Goldstein, Proc. Natl. Acad. Sci. U.S.A. 103, 8315 (2006).

[27] S. Michelin and E. Lauga, Phys. Fluids 23, 101901 (2011).

[28] D. Tam and A. E. Hosoi, Proc. Natl. Acad. Sci. U.S.A. 108, 1001 (2011).

[29] A. J. T. M. Mathijssen, F. Guzmán-Lastra, A. Kaiser, and H. Löwen, Phys. Rev. Lett. 121, 248101 (2018).

[30] D. Papavassiliou and G. P. Alexander, Europhys. Lett. 110, 44001 (2015).

[31] H. Shum and J. M. Yeomans, Phys. Rev. Fluids 2, 113101 (2017).

[32] P. Mueller and J.-L. Thiffeault, Phys. Rev. Fluids 2, 013103 (2017).

[33] L. Vaccari, M. Molaei, R. L. Leheny, and K. J. Stebe, Soft Matter 14, 5643 (2018).

[34] A. Purushothaman and S. P. Thampi, Soft Matter 17, 3380 (2021).

[35] C. J. Ingham, O. Kalisman, A. Finkelshtein, and E. Ben-Jacob, Proc. Natl. Acad. Sci. U.S.A. 108, 19731 (2011).

[36] I. Tuval, L. Cisneros, C. Dombrowski, C. W. Wolgemuth, J. O. Kessler, and R. E. Goldstein, Proc. Natl. Acad. Sci. U.S.A. 102, 2277 (2005).

[37] A. J. T. M. Mathijssen, R. Jeanneret, and M. Polin, Phys. Rev. Fluids 3, 033103 (2018).

[38] R. Dreyfus, J. Baudry, M. L. Roper, M. Fermigier, H. A. Stone, and J. Bibette, Nature (London) 437, 862 (2005).

[39] J. R. Howse, R. A. L. Jones, A. J. Ryan, T. Gough, R. Vafabakhsh, and R. Golestanian, Phys. Rev. Lett. 99, 048102 (2007).

[40] C. Peng, I. Lazo, S. V. Shiyanovskii, and O. D. Lavrentovich, Phys. Rev. E 90, 051002(R) (2014).

[41] D. Nishiguchi and M. Sano, Phys. Rev. E 92, 052309 (2015).

[42] C. Bechinger, R. Di Leonardo, H. Löwen, C. Reichhardt, G. Volpe, and G. Volpe, Rev. Mod. Phys. 88, 045006 (2016).

[43] J. Elgeti, R. G. Winkler, and G. Gompper, Rep. Prog. Phys. 78, 056601 (2015).
[44] A. Zöttl and H. Stark, J. Phys. Condens. Matter 28, 253001 (2016).

[45] A. I. Campbell, S. J. Ebbens, P. Illien, and R. Golestanian, Nat. Commun. 10, 1 (2019).

[46] B. J. Nelson, I. K. Kaliakatsos, and J. J. Abbott, Annu. Rev. Biomed. Eng. 12, 55 (2010).

[47] G. Tiwari, R. Tiwari, B. Sriwastawa, L. Bhati, S. Pandey, P. Pandey, and S. K. Bannerjee, Int. J. Pharm. Invest. 2, 2 (2012).

[48] S. Wilhelm, A. J. Tavares, Q. Dai, S. Ohta, J. Audet, H. F. Dvorak, and W. C. Chan, Nat. Rev. Mater. 1, 16014 (2016).

[49] S. Shen, Y. Wu, Y. Liu, and D. Wu, Int. J. Nanomed. 12, 4085 (2017).

[50] D. Xu, Y. Wang, C. Liang, Y. You, S. Sanchez, and X. Ma, Small 16, 1902464 (2020).

[51] Y. Alapan, O. Yasa, B. Yigit, I. C. Yasa, P. Erkoc, and M. Sitti, Annu. Rev. Control Robot. Autonom. Sys. 2, 205 (2019).

[52] P. Erkoc, I. C. Yasa, H. Ceylan, O. Yasa, Y. Alapan, and M. Sitti, Adv. Therap. 2, 1800064 (2019).

[53] A. Daddi-Moussa-Ider, M. Lisicki, and A. J. T. M. Mathijssen, Phys. Rev. Applied 14, 024071 (2020).

[54] L. Yang and L. Zhang, Annu. Rev. Control Robot. Autonom. Sys. 4, 509(2020).

[55] S. Thutupalli, R. Seemann, and S. Herminghaus, New J. Phys. 13, 073021 (2011).

[56] C. C. Maass, C. Krüger, S. Herminghaus, and C. Bahr, Annu. Rev. Condens. Matter Phys. 7, 171 (2016).

[57] S. Thutupalli and S. Herminghaus, Eur. Phys. J. E 36, 91 (2013).

[58] Z. Izri, M. N. van der Linden, S. Michelin, and O. Dauchot, Phys. Rev. Lett. 113, 248302 (2014).

[59] C. Krueger, C. Bahr, S. Herminghaus, and C. C. Maass, Eur. Phys. J. E 39, 64 (2016).

[60] C. Krüger, G. Klös, C. Bahr, and C. C. Maass, Phys. Rev. Lett. 117, 048003 (2016).

[61] C. Jin, C. Krüger, and C. C. Maass, Proc. Natl. Acad. Sci. U.S.A. 114, 5089 (2017).

[62] S. Thutupalli, D. Geyer, R. Singh, R. Adhikari, and H. A. Stone, Proc. Natl. Acad. Sci. U.S.A. 115, 5403 (2018).

[63] C. Jin, B. V. Hokmabad, K. A. Baldwin, and C. C. Maass, J. Phys. Condens. Matter 30, 054003 (2018).

[64] C. de Blois, M. Reyssat, S. Michelin, and O. Dauchot, Phys. Rev. Fluids 4, 054001 (2019).

[65] C. Stamatopoulos, A. Milionis, N. Ackerl, M. Donati, P. Leudet de la Vallée, P. Rudolf von Rohr, and D. Poulikakos, ACS Nano 14, 12895 (2020).

[66] B. V. Hokmabad, R. Dey, M. Jalaal, D. Mohanty, M. Almukambetova, K. A. Baldwin, D. Lohse, and C. C. Maass, Phys. Rev. X 11, 011043 (2021).

[67] K. Lippera, M. Morozov, M. Benzaquen, and S. Michelin, J. Fluid Mech. 886, A17 (2020).

[68] B. V. Hokmabad, S. Saha, J. A. Canalejo, R. Golestanian, and C. C. Maass, arXiv:2012.05170.

[69] See Supplemental Material at http://link.aps.org/ supplemental/10.1103/PhysRevLett.127.088006 for experimental methods, theoretical derivations, a Supplemental Figure, 3 Videos, and additional references [70-84].

[70] D. Qin, Y. Xia, and G. M. Whitesides, Nat. Protoc. 5, 491 (2010). 
[71] T. Thorsen, R. W. Roberts, F. H. Arnold, and S. R. Quake, Phys. Rev. Lett. 86, 4163 (2001).

[72] J. C. Crocker and D. G. Grier, J. Colloid Interface Sci. 179, 298 (1996).

[73] R. Jeanneret, D. O. Pushkin, and M. Polin, Phys. Rev. Lett. 123, 248102 (2019).

[74] R.-Y. Tsay and S. Weinbaum, J. Fluid Mech. 226, 125 (1991).

[75] R. E. Pepper, M. Roper, S. Ryu, P. Matsudaira, and H. A. Stone, J. R. Soc. Interface 7, 851 (2010).

[76] D. O. Pushkin and M. A. Bees, in Biophysics of Infection. Advances in Experimental Medicine and Biology, edited by M. Leake (Springer, New York, 2016), Vol. 915, pp. 193-205.

[77] H. Nganguia and O. S. Pak, J. Fluid Mech. 855, 554 (2018).

[78] H. Nganguia, L. Zhu, D. Palaniappan, and O. S. Pak, Phys. Rev. E 101, 063105 (2020).

[79] S. Whitaker, Transp. Porous Media 1, 3 (1986).

[80] W. Gilpin, V. N. Prakash, and M. Prakash, Nat. Phys. 13, 380 (2017).

[81] C. Liu, C. Zhou, W. Wang, and H. P. Zhang, Phys. Rev. Lett. 117, 198001 (2016).

[82] J. D. Weeks, D. Chandler, and H. C. Andersen, J. Chem. Phys. 54, 5237 (1971).
[83] I. Eames and M.E. McIntyre, Math. Proc. Cambridge Philos. Soc. 126, 171 (1999).

[84] J. O. Dabiri, J. Exp. Biol. 208, 3519 (2005).

[85] S. Herminghaus, C. C. Maass, C. Krüger, S. Thutupalli, L. Goehring, and C. Bahr, Soft Matter 10, 7008 (2014).

[86] M. J. Lighthill, Commun. Pure Appl. Math. 5, 109 (1952).

[87] J. R. Blake, J. Fluid Mech. 46, 199 (1971).

[88] H. C. Brinkman, Physica 13, 447 (1947).

[89] A. J. T. M. Mathijssen, D. O. Pushkin, and J. M. Yeomans, J. Fluid Mech. 773, 498 (2015).

[90] J. Simmchen, J. Katuri, W. E. Uspal, M. N. Popescu, M. Tasinkevych, and S. Sánchez, Nat. Commun. 7, 10598 (2016).

[91] A. Zöttl and H. Stark, Phys. Rev. Lett. 112, 118101 (2014).

[92] H. Wioland, E. Lushi, and R. E. Goldstein, New J. Phys. 18, 075002 (2016).

[93] L. Vidakovic, P. K. Singh, R. Hartmann, C. D. Nadell, and K. Drescher, Nat. Rev. Microbiol. 3, 26 (2018).

[94] T. Bhattacharjee and S. S. Datta, Nat. Commun. 10, 2075 (2019).

[95] Q. Roveillo, J. Dervaux, Y. Wang, F. Rouyer, D. Zanchi, L. Seuront, and F. Elias, J. R. Soc. Interface 17, 20200077 (2020). 